DOI: $10.12957 /$ demetra.2015.15112

\title{
Associação entre a percepção materna do estado nutricional do filho e a qualidade da dieta de escolares
}

\section{Association between maternal perception of children's nutritional status and quality of diet of schoolchildren}

\author{
Luana da Silva Baptista Arpini' \\ Geovane Carlos Barbosa ${ }^{2}$ \\ Gláucia Figueiredo Justo $^{3}$ \\ Luciane Bresciani Salaroli4 \\ Maria del Carmen Bisi Molina ${ }^{4}$ \\ - Secretaria de Saúde do Estado do Espírito \\ Santo, Hospital Estadual Infantil Nossa Senhora \\ da Glória. Cariacica-ES, Brasil. \\ ${ }^{2}$ Faculdade do Centro Leste, Departamento de \\ Engenharia Biomédica. Serra-ES, Brasil. \\ ${ }^{3}$ Universidade Federal do Rio de Janeiro, \\ Instituto de Estudos em Saúde Coletiva. Rio de \\ Janeiro-RJ, Brasil. \\ ${ }^{4}$ Universidade Federal do Espírito Santo, \\ Departamento de Educação Integrada em Saúde, \\ Curso de Nutrição. Vitória-ES, Brasil. \\ Correspondência / Correspondence \\ Luana da Silva Baptista Arpini \\ E-mail: luanaarpini@hotmail.com
}

\section{Resumo}

Objetivo: analisar a associação entre a percepção materna do estado nutricional do filho e a qualidade da dieta de escolares. Métodos: Abordagem seccional, com 1.788 escolares (1.272 de região urbana e 516 de região rural). Foram coletados dados sociodemográficos, antropométricos e de alimentação. Utilizou-se o Índice ALES para avaliar a qualidade da dieta. O teste qui-quadrado foi utilizado para determinar as diferenças de proporções entre os grupos e o modelo de regressão logística para ajustar as variáveis associadas. Resultados: Os escolares apresentaram maiores percentuais de baixa qualidade da dieta tanto na região urbana $(40,4 \%)$, como na rural $(38,6 \%)$. Concordância da percepção materna com o diagnóstico nutricional infantil não influenciou a qualidade global da dieta. Raça/cor (preto/pardo), menor nível de classe socioeconômica (C e D+E) e escolaridade materna baixa ( $<3$ anos de estudo) e intermediária (4-10 anos) aumentaram as chances de baixa qualidade da dieta do escolar. Conclusão: Não foi encontrada associação entre a alimentação infantil e a concordância da percepção materna com o diagnóstico do estado nutricional. Todavia, os determinantes socioeconômicos influenciaram diretamente na qualidade da dieta dos escolares.

Palavras-chave: Percepção. Estado Nutricional. Alimentação. Dieta. Criança. Saúde Escolar. 


\section{Abstract}

Objective: To assess the association between maternal perception of children's nutritional status and the quality of the diet of schoolchildren from urban and rural regions. Methods: A crosssectional approach, with 1,788 schoolchildren (1,272 urban and 516 rural). Sociodemographic, anthropometric and feeding data were obtained. We used the ALES Index to assess diet quality. The chi-square test was used to determine differences in proportions between groups and logistic regression model to adjust associated variables. Results: Schoolchildren had higher percentages of low diet quality in urban (40.4\%) and in rural (38.6\%). Agreement with maternal perception of infant nutritional diagnosis did not influence the overall quality of the diet. Race/skin color (black/brown), lower socioeconomic class (C and D + E) and low ( $<3$ years of study) and intermediate (4-10 years) maternal educational level increased the odds of poor diet quality of schoolchildren. Conclusion: There was no association between the child's feeding and the concordance of the maternal perception with the child's nutritional status's diagnosis. However, the socioeconomic determinants directly influenced the quality of the diet of schoolchildren.

Key words: Perception. Nutritional Status. Feeding. Diet. Child. School Health.

\section{Introdução}

A qualidade da dieta é fundamental para o crescimento e desenvolvimento adequado da criança. ${ }^{1} \mathrm{Na}$ faixa etária escolar, em especial, é importante incentivar os pais a estimularem comportamentos alimentares adequados, uma vez que nessa idade há aumento da independência na escolha e consumo dos alimentos. De acordo com estudo de Kranz et al., ${ }^{2}$ a cada um ano de incremento na idade, há uma perda de aproximadamente dois pontos no índice da qualidade da dieta, ou seja, à medida que aumenta a idade da criança, diminui a qualidade global da dieta que esta apresenta.

Apesar das inúmeras tentativas de incentivo aos hábitos alimentares saudáveis, a alimentação dos escolares não tem atingido as recomendações. Dados da Pesquisa Nacional de Saúde do Escolar $(\mathrm{PeNSE})^{3}$ revelam consumo regular dos marcadores de alimentação não saudável (refrigerantes, 
salgadinhos, doces) e consumo inferior ao recomendado de alimentação saudável (frutas, hortaliças, fibras) pelos adolescentes brasileiros, apontando a necessidade de ações de educação e promoção de saúde dirigidas à população mais jovem.

Pesquisas realizadas nas cidades brasileiras de Ouro Preto-MG, ${ }^{4}$ Vitória-ES ${ }^{5}$ e Florianópolis-SC, ${ }^{6}$ com crianças e adolescentes com idade entre seis e 14 anos, também encontraram baixos escores de qualidade da dieta, refletindo um padrão alimentar em desacordo com as recomendações.

Coorte prospectiva de crianças e adolescentes na faixa etária de sete a 15 anos do Reino Unido confirma que um padrão alimentar com alta densidade energética, rico em gordura e pobre em fibras está associado a maiores níveis de obesidade e excesso de adiposidade na infância e adolescência, independentemente da atividade física. ${ }^{7}$ A qualidade da dieta infantil pode ser influenciada por uma ampla variedade de fatores, dentre os quais os biológicos, comportamentais, as preferências e as práticas alimentares familiares, a disponibilidade de alimentos, determinantes socioeconômicos, crenças culturais e a percepção dos pais. ${ }^{1}$

Considerando que o comportamento alimentar infantil pode ser modificado de acordo com o ambiente no qual a criança está inserida, a percepção dos pais pode ser uma ferramenta crucial para determinar a qualidade da dieta, sendo capaz de influenciar positiva ou negativamente na alimentação ofertada. ${ }^{1}$

A percepção materna do peso corporal do filho influencia nas práticas de controle alimentar infantil, mediadas ou não pela preocupação materna com o peso da criança. ${ }^{8}$ Portanto, a percepção materna, quando concordante com o estado nutricional do filho, pode propiciar modificações alimentares precoces. ${ }^{5,8}$ Por outro lado, a discordância desses pode negligenciar comportamentos e padrões alimentares inadequados como, por exemplo, a pressão para que os filhos comam maiores porções do que seriam adequadas para a idade -promovendo a superalimentação e o ganho de peso. ${ }^{8,9} \mathrm{E}$ ainda, comprometer a eficácia do tratamento nos distúrbios nutricionais. ${ }^{8}$

Além disso, é possível que a percepção materna do estado nutricional do filho esteja relacionada à qualidade global da dieta ofertada para a criança, sobretudo na faixa etária escolar, em que se encontram as maiores prevalências de obesidade. ${ }^{8}$ Entretanto, em revisão sistemática de literatura sobre o tema, publicada recentemente, não foram encontrados estudos que tenham avaliado a qualidade da alimentação consumida pela criança, reforçando a necessidade de investigações que avaliem a relação entre a percepção materna do estado nutricional do filho e a qualidade da dieta infantil, utilizando métodos de mensuração alimentar adequados. ${ }^{8}$ Desta forma, o objetivo deste estudo é analisar a associação entre a percepção materna do estado nutricional do filho e a qualidade da alimentação de escolares de duas regiões distintas. 


\section{Métodos}

Estudo descritivo, de abordagem seccional, realizado em 2013 e conduzido a partir da análise dos dados obtidos no âmbito dos projetos "Saúdes Vitória” e "Saúdes Santa Maria”, ambos de base escolar, desenvolvidos com o objetivo de investigar os aspectos relacionados à nutrição e saúde de escolares matriculados no primeiro ciclo do Ensino Fundamental de escolas públicas e privadas dos municípios de Vitória e Santa Maria de Jetibá, no estado do Espírito Santo, Brasil.

Foram incluídas as crianças de ambos os sexos, na faixa etária de sete a 10 anos completos no dia da coleta de dados, regularmente matriculadas, que apresentavam o Termo de Consentimento Livre e Esclarecido (TCLE) assinado pelo responsável e que não apresentaram questionários com "não resposta" às variáveis associadas (percepção materna do peso do filho, estado nutricional e qualidade da dieta infantil), perfazendo uma amostra composta por 1.788 crianças, sendo 1.272 da região urbana e 516 da região rural.

Compreende-se por população urbana os escolares que participaram do estudo "Saúdes Vitória", realizado em 2007 na capital do estado do Espírito Santo, considerada 100\% urbanizada, utilizando amostra representativa da população da cidade para faixa etária, sexo e situação de matrícula, tanto na rede pública quanto privada de ensino. A população rural são os participantes do estudo "Saúdes Santa Maria”, conduzido em 2009 em município rural colonizado por "Pomeranos", que tentam manter a cultura e tradição de suas origens como, por exemplo, a alimentação e o dialeto, cuja amostra também foi representativa da população escolar.

O processo de amostragem adotado foi do tipo probabilístico (aleatório estratificado) por conglomerado de triplo estágio em Vitória, ${ }^{10}$ e duplo estágio em Santa Maria de Jetibá, ${ }^{11}$ planejados de maneira que ambos os municípios fossem completamente abrangidos geograficamente segundo suas zonas escolares, sexo e faixa etária de interesse. Os dados socioeconômicos, biológicos e de hábitos de vida foram obtidos a partir de um questionário estruturado respondido pelas mães. Para os indivíduos da região rural, os questionários poderiam ser respondidos no idioma pomerano, respeitando e não constrangendo no momento da entrevista.

Informações sobre consumo alimentar foram obtidas a partir de um questionário de frequência alimentar (QFA) com 18 itens alimentares. Após estudo piloto ${ }^{12}$ realizado antes da coleta de dados, foi verificada a necessidade de adaptação do QFA aplicado à população rural, devido à cultura alimentar específica apresentada por essa população.

Para avaliação da qualidade da dieta, foi utilizada a proposta de Molina et al., ${ }^{5}$ que leva em consideração a frequência de consumo de 15 itens alimentares e a prática de realizar a primeira refeição matinal (desjejum), denominado Índice ALES. Este índice foi desenvolvido para a população de escolares do estado do Espírito Santo e resulta em um escore de resumo, que permite avaliar a qualidade global da dieta de crianças de 7-10 anos, classificadas de acordo 
com a seguinte pontuação: $<3=$ baixa qualidade; entre $3 \geq$ e $<6=$ qualidade intermediária; e valores $>6=$ boa qualidade. ${ }^{5}$

Dentre as variáveis associadas, a concordância da percepção materna do estado nutricional do filho foi obtida através da pergunta: "Como a senhora acha que seu filho está?", cujas opções de resposta correspondiam a "abaixo do peso", "peso adequado", "acima do peso" ou "muito acima do peso", posteriormente associada ao estado nutricional infantil e recategorizada em: concordante (quando a percepção da mãe corresponde ao estado nutricional diagnosticado do filho) e discordante (quando a mãe subestima ou superestima o estado nutricional de seu filho). O IMC materno foi estimado usando o peso e a altura referidos pela mãe e classificado de acordo com a Organização Mundial da Saúde (OMS); ${ }^{13}$ a escolaridade materna correspondeu ao último ano de estudo concluído pela mãe, categorizada em $\leq 3,4-10, \geq 11$ anos; o relato materno sobre a presença ou não de preocupação com o peso do filho e a classe socioeconômica obtida através do escore de pontuações proposto pela Associação Brasileira de Empresas de Pesquisa (ABEP) ${ }^{14}$ e reclassificada em $\mathrm{A}+\mathrm{B}, \mathrm{C}$ e $\mathrm{D}+\mathrm{E}$.

As variáveis infantis foram: sexo, idade (obtida pela subtração do ano da coleta de dados com o de nascimento da criança), raça/cor (classificada por 2 entrevistadores independentes e dicotomizada em "branco" e "preto/pardo") e estado nutricional (obtido pelo cálculo do Índice de massa corporal - IMC a partir das medidas de peso e estatura, e classificado de acordo com os pontos de corte propostos por Onis et al. ${ }^{15}$ ).

A antropometria foi realizada nas escolas por profissionais treinados, utilizando os procedimentos padronizados, proposto pela OMS. ${ }^{16} \mathrm{O}$ peso foi aferido em quilogramas, com precisão de 100 gramas utilizando balanças Tanita ${ }^{\circledR}$ Family BWF (Tanita, Illinois, USA). Durante a aferição, as crianças eram pesadas sem sapatos e com o mínimo de roupas possível, dispostos no centro da balança, com os braços estendidos ao lado do corpo e o olhar fixo a sua frente.

A estatura foi medida em centímetros, com precisão de um milímetro utilizando estadiômetro portátil da marca Seca ${ }^{\circledR}$ modelo 206 (Seca, Hamburgo, BRD). O estadiômetro foi afixado na parede lisa e sem rodapé. No momento da medida, as crianças estavam descalças, com os cabelos soltos e com a cabeça, nádegas e calcanhares juntos à parede e olhar fixo a sua frente.

Para análise dos dados, os bancos foram unificados cuidadosamente após revisão e conferência com os questionários, compilados no programa Microsoft ${ }^{\circledR}$ Office Excel 2007, e posteriormente transportados para análise no software IBM $^{\circledR}$ SPSS For Windows versão 20.0. Estatísticas descritivas foram calculadas para as variáveis de interesse. Os testes qui-quadrado e exato de Fisher foram utilizados para determinar as diferenças de proporções entre os grupos $(\mathrm{p}<0,10)$. Para avaliar a associação entre a percepção materna do estado nutricional do filho com a qualidade da dieta infantil, bem como ajustar com os fatores socioeconômicos, as variáveis que apresentaram significância de $10 \%$ na análise univariada, foram incluídas no modelo de regressão logística multinomial. 
Quanto às considerações éticas, foram respeitados os dispositivos da Resolução no 196/96 do Conselho Nacional de Saúde, sendo o estudo conduzido após aprovação pelo Comitê de Ética em Pesquisa da Universidade Federal do Espírito Santo e pelas autoridades locais de cada município.

\section{Resultados}

Na tabela 1, estão apresentadas as características sociodemográficas da população estudada segundo região de residência.

Tabela 1. Caracterização da amostra de escolares de 7-10 anos, segundo região de residência. Vitória (2007) e Santa Maria de Jetibá (2009).

\begin{tabular}{|c|c|c|c|c|c|c|}
\hline \multirow{3}{*}{ Variável } & \multirow{2}{*}{\multicolumn{2}{|c|}{ Total }} & \multicolumn{4}{|c|}{ Região de residência do escolar } \\
\hline & & & \multicolumn{2}{|c|}{ Urbana } & \multicolumn{2}{|c|}{ Rural } \\
\hline & $\mathrm{n}$ & $\%$ & $\mathrm{n}$ & $\%$ & $\mathrm{n}$ & $\%$ \\
\hline \multicolumn{7}{|l|}{ Sexo } \\
\hline Masculino & 802 & 44,9 & 532 & 41,8 & 270 & 52,3 \\
\hline Feminino & 986 & 55,1 & 740 & 58,2 & 246 & 47,7 \\
\hline \multicolumn{7}{|l|}{ Idade } \\
\hline 7 & 404 & 22,6 & 249 & 19,6 & 155 & 30,0 \\
\hline 8 & 495 & 27,7 & 358 & 28,1 & 137 & 26,6 \\
\hline 9 & 481 & 26,9 & 356 & 28,0 & 125 & 24,2 \\
\hline 10 & 408 & 22,8 & 309 & 24,3 & 99 & 19,2 \\
\hline \multicolumn{7}{|l|}{ Estado nutricional } \\
\hline Magreza & 92 & 5,1 & 73 & 5,7 & 19 & 3,7 \\
\hline Eutrófico & 1333 & 74,6 & 902 & 70,9 & 431 & 83,5 \\
\hline Sobrepeso & 221 & 12,4 & 182 & 14,3 & 39 & 7,6 \\
\hline Obeso & 27 & 7,9 & 115 & 9,0 & 27 & 5,2 \\
\hline \multicolumn{7}{|l|}{ Raça/Cor } \\
\hline Branco & 804 & 47,3 & 418 & 32,9 & 386 & 81,8 \\
\hline Preto/Pardo & 897 & 52,7 & 811 & 63,8 & 86 & 18,2 \\
\hline
\end{tabular}


Qualidade da dieta

Baixa

Média

Boa

Classe socioeconômica
$\mathrm{A}+\mathrm{B}$
C
$\mathrm{D}+\mathrm{E}$

Idade materna (anos)

$$
\begin{aligned}
& \leq 30 \\
& >30
\end{aligned}
$$

1233

Escolaridade materna (anos)

$$
\begin{aligned}
& \leq 3 \\
& 4-10 \\
& \geq 11
\end{aligned}
$$

IMC materno

$$
\text { Magreza }
$$

Eutrofia

Sobrepeso

Obesidade

Concordância da PM

Concordante

Discordante

Preocupação materna

\begin{tabular}{rrrrrrr} 
Sim & 996 & 58,2 & 725 & 60,5 & 271 & 53,0 \\
Não & 714 & 41,8 & 474 & 39,5 & 240 & 47,0 \\
\hline Total & 1788 & 100 & 1272 & 71,1 & 516 & $\mathbf{2 8 , 9}$ \\
\hline
\end{tabular}

$\begin{array}{llllll}713 & 39,9 & 514 & 40,4 & 199 & 38,6 \\ 452 & 25,3 & 309 & 24,3 & 143 & 27,7 \\ 623 & 34,8 & 449 & 35,3 & 174 & 33,7\end{array}$

$\begin{array}{llllll}282 & 17,9 & 262 & 23,5 & 20 & 4,3 \\ 659 & 41,8 & 392 & 35,2 & 267 & 57,7 \\ 637 & 40,4 & 461 & 41,3 & 176 & 38,0\end{array}$

$\begin{array}{llllll}513 & 29,4 & 358 & 29,0 & 155 & 30,3\end{array}$

$\begin{array}{llllll}233 & 70,6 & 877 & 71,0 & 356 & 69,7\end{array}$

$\begin{array}{cccccc}59 & 3,4 & 50 & 4,0 & 9 & 1,8 \\ 935 & 53,3 & 521 & 41,8 & 414 & 81,5 \\ 759 & 43,3 & 674 & 54,1 & 85 & 16,7\end{array}$

$3,1 \quad 38$

3,3 $\quad 10$

2,7

814

53,3

627

$54,1 \quad 187$

50,7

465

30,4

353

30,5

11230,4

201

13,2

141

12,2

60

16,3

$\begin{array}{llllll}1087 & 60,8 & 741 & 58,3 & 346 & 67,2 \\ 700 & 39,2 & 531 & 41,7 & 169 & 32,8\end{array}$

$41,7 \quad 169$

32,8 
A região urbana apresentou maiores percentuais de escolares do sexo feminino (58,2\%), com idade de 8-9 anos (28,0\%), não brancos (63,8\%), de classe socioeconômica mais baixa (41,3\%) e escolaridade materna mais alta $(54,1 \%)$. Já a região rural é composta, em sua maioria, por uma população branca $(81,8 \%)$, de escolares mais jovens e do sexo masculino (52,3\%), classe socioeconômica e escolaridade materna intermediárias (57,7\% e 81,5\%, respectivamente), segundo a tabela 1 .

Quanto ao estado nutricional infantil, os resultados mostram maiores prevalências tanto de excesso de peso (sobrepeso e obesidade) quanto de magreza, nos escolares urbanos. Ainda assim, evidencia-se que ambas as regiões apresentaram muito mais escolares com excesso de peso do que com magreza (23,3\% versus $5,7 \%$ na região urbana e $12,8 \%$ e 3,7\% na rural, respectivamente), conforme a tabela 1. O mesmo aconteceu para as mães, que apresentaram prevalências de excesso de peso superiores a $40 \%$ (42,7\% na região urbana e $46,7 \%$ na rural).

Tanto na região urbana, quanto na rural, a maioria das mães apresentou maiores percentuais de percepção do estado nutricional de seus filhos, correspondente ao diagnosticado, e referiram preocupação com o peso atual ou futuro de seus filhos. No entanto, observa-se maior concordância naquelas residentes na região rural $(67,2 \%$ versus $60,8 \%)$ e percentuais superiores de preocupação das mães da região urbana (60,5\% versus 53,0\%), ainda segundo a tabela 1 .

Na tabela 2, são apresentados os resultados da análise univariada entre as variáveis e qualidade da dieta de escolares de 7-10 anos de regiões urbana e rural.

Tabela 2. Diferença de proporções observadas entre a qualidade da dieta e fatores associados em escolares de 7-10 anos. Vitória (2007) e Santa Maria de Jetibá (2009).

\begin{tabular}{|c|c|c|c|c|c|c|c|c|c|}
\hline \multirow{3}{*}{ Variável } & \multirow{2}{*}{\multicolumn{2}{|c|}{ Total }} & \multicolumn{6}{|c|}{ Qualidade da dieta } & \multirow{3}{*}{$p$} \\
\hline & & & \multicolumn{2}{|c|}{ Baixa } & \multicolumn{2}{|c|}{ Média } & \multicolumn{2}{|c|}{ Boa } & \\
\hline & $\mathrm{n}$ & $\%$ & $\mathrm{n}$ & $\%$ & $\mathrm{n}$ & $\%$ & $\mathrm{n}$ & $\%$ & \\
\hline \multicolumn{10}{|l|}{ Sexo } \\
\hline Masculino & 802 & 44,9 & 299 & 41,9 & 208 & 46,0 & 295 & 47,4 & \multirow{2}{*}{0,118} \\
\hline Feminino & 986 & 55,1 & 414 & 58,1 & 244 & 54,0 & 328 & 52,6 & \\
\hline \multicolumn{10}{|l|}{ Idade (anos) } \\
\hline 7 & 404 & 22,6 & 162 & 22,7 & 115 & 25,4 & 127 & 20,4 & \multirow{4}{*}{0,192} \\
\hline 8 & 495 & 27,7 & 190 & 26,6 & 126 & 27,9 & 179 & 28,7 & \\
\hline 9 & 481 & 26,9 & 182 & 25,5 & 115 & 25,4 & 184 & 29,5 & \\
\hline 10 & 408 & 22,8 & 179 & 25,1 & 96 & 21,2 & 133 & 21,3 & \\
\hline
\end{tabular}


Estado nutricional

$\begin{array}{lccccccccc}\text { Magreza } & 92 & 5,1 & 43 & 6,0 & 18 & 4,0 & 31 & 5,0 & \\ \text { Eutrófico } & 1333 & 74,6 & 533 & 74,8 & 342 & 75,7 & 458 & 73,5 & \\ \text { Sobrepeso } & 221 & 12,4 & 79 & 11,1 & 62 & 19,1 & 80 & 12,8 & 0,466 \\ \text { Obeso } & 142 & 7,9 & 58 & 8,1 & 30 & 13,7 & 54 & 8,7 & \end{array}$

Raça/cor

Branco

$\begin{array}{lllllllll}804 & 47,3 & 282 & 41,5 & 212 & 49,5 & 310 & 52,2 & \\ 897 & 52,7 & 397 & 58,5 & 216 & 50,5 & 284 & 47,8 & <0,001\end{array}$

Região de

residência

Urbana

1272

$\begin{array}{lllllll}71,1 & 514 & 72,1 & 309 & 68,4 & 449 & 72,1\end{array}$

Rural

$\begin{array}{llllllll}516 & 28,9 & 199 & 27,9 & 143 & 31,6 & 174 & 27,9\end{array}$

0,321

Classe

socioeconômica

$\begin{array}{lrrrrrrrrr}\text { A+B } & 282 & 17,9 & 76 & 12,3 & 70 & 17,5 & 136 & 24,2 & \\ \text { C } & 659 & 41,8 & 262 & 42,5 & 165 & 41,1 & 232 & 41,4 & <0,001 \\ \text { D+E } & 637 & 40,4 & 278 & 45,1 & 166 & 41,4 & 193 & 34,4 & \end{array}$

Idade materna (anos)

$$
\begin{array}{lcccccccc}
\leq 30 & 513 & 29,4 & 217 & 31,5 & 136 & 30,8 & 160 & 26,0 \\
>30 & 1233 & 70,6 & 472 & 68,5 & 305 & 69,2 & 456 & 74,0
\end{array}
$$

Escolaridade materna (anos)

$\begin{array}{lccccccccc}\leq 3 & 59 & 3,4 & 36 & 5,1 & 11 & 2,5 & 12 & 2,0 & \\ 4-10 & 936 & 53,4 & 421 & 60,1 & 229 & 51,6 & 286 & 46,9 & <0,001 \\ \geq 11 & 759 & 43,3 & 243 & 34,7 & 204 & 45,9 & 312 & 51,1 & \end{array}$


IMC materno

$\begin{array}{lcccccccc}\text { Magreza } & 48 & 3,1 & 18 & 3,0 & 15 & 3,9 & 15 & 2,8 \\ \text { Eutrofia } & 814 & 53,3 & 309 & 51,2 & 198 & 52,1 & 307 & 56,3 \\ \text { Sobrepeso } & 465 & 30,4 & 179 & 29,7 & 117 & 30,8 & 169 & 31,0 \\ \text { Obesidade } & 201 & 13,2 & 97 & 16,1 & 50 & 13,2 & 54 & 9,9\end{array}$

Concordância

da PM

Discordante

$700 \quad 39,2 \quad 299$

$41,9 \quad 176$

$39,0 \quad 225 \quad 36,1$

0,094

Preocupação

materna

Sim

99658

Não

$714 \quad 41,8$

41,8

$60,7 \quad 25$

56,9

333

56,4

$266 \quad 39,3 \quad 191 \quad 43,1 \quad 257 \quad 43,6$

0,245

Total

A variável "concordância da percepção materna" apresentou associação estatisticamente significativa com a qualidade da dieta dos escolares $(p=0,094)$, permanecendo as maiores prevalências nas três classificações do Índice ALES (tabela 2). As variáveis "raça/cor" ( $p=<0,001)$, "classe socioeconômica" ( $p=<0,001)$, "idade materna" $(p=0,068)$, "IMC materno" $(p=0,085)$ e "escolaridade materna" ( $\mathrm{p}=<0,001)$ também apresentaram associação significativa com a qualidade global da dieta. Os escolares pretos/pardos, de classe socioeconômica mais baixa e escolaridade materna intermediária, estiveram associados ao nível mais baixo de qualidade da dieta do escolar. E ser branco, de classe média e ter mãe com maior escolaridade foi relacionado ao melhor índice de qualidade da dieta do escolar (tabela 2).

Todas as variáveis supracitadas foram inseridas no modelo de regressão logística multinomial para ajuste. Todavia, a associação entre a concordância da percepção materna e a qualidade da dieta do escolar não permaneceu. As variáveis que se mantiveram associadas à qualidade da dieta, após ajuste, foram os determinantes socioeconômicos: raça/cor, classe socioeconômica e escolaridade materna (tabela 3). 
Tabela 3. Fatores associados à qualidade da dieta de escolares de 7-10 anos: odds ratio ajustado (OR) com respectivo intervalo de confiança (IC95\%). Vitória (2007) e Santa Maria de Jetibá (2009).

\begin{tabular}{|c|c|c|c|c|}
\hline \multirow{3}{*}{ Variável } & \multicolumn{4}{|c|}{ Qualidade da dieta } \\
\hline & \multicolumn{2}{|l|}{ Baixa } & \multicolumn{2}{|l|}{ Média } \\
\hline & OR (IC95\%) & $\mathrm{p}$ & OR (IC95\%) & $\mathrm{p}$ \\
\hline \multicolumn{5}{|l|}{ Raça/cor } \\
\hline Branco & 1,000 & & 1,000 & \\
\hline Preto/pardo & $1,400(1,090-1,797)$ & 0,008 & $1,030(0,781-1,359)$ & 0,835 \\
\hline \multicolumn{5}{|l|}{ Classe } \\
\hline \multicolumn{5}{|l|}{ socioeconômica } \\
\hline $\mathrm{A}+\mathrm{B}$ & 1,000 & & 1,000 & \\
\hline $\mathrm{C}$ & $1,505(1,046-2,166)$ & 0,028 & $1,343(0,912-1,978)$ & 0,135 \\
\hline $\mathrm{D}+\mathrm{E}$ & $1,507(1,009-2,250)$ & 0,045 & $1,700(1,106-2,611)$ & 0,015 \\
\hline \multicolumn{5}{|l|}{$\begin{array}{l}\text { Escolaridade } \\
\text { materna (anos) }\end{array}$} \\
\hline$\leq 3$ & $2,979(1,423-6,239)$ & 0,004 & $1,070(0,435-2,635)$ & 0,882 \\
\hline $4-10$ & $1,658(1,252-2,194)$ & $<0,001$ & $0,962(0,707-1,309)$ & 0,807 \\
\hline$\geq 11$ & 1,000 & & 1,000 & \\
\hline
\end{tabular}

*Categoria de referência: boa qualidade da dieta.

Ser preto/pardo $(\mathrm{p}=0,008 ; \mathrm{OR}=1,400 ; \mathrm{IC} 95 \%=1,090-1,797)$ aumentou em $40 \%$ a chance de o escolar apresentar dieta de baixa qualidade. As crianças das classes socioeconômicas média $(\mathrm{p}=0,028 ; \mathrm{OR}=1,505 ; \mathrm{IC} 95 \%=1,046-2,166)$ e baixa $(\mathrm{p}=0,045 ; \mathrm{OR}=1,507 ; \mathrm{IC} 95 \%=1,009-2,250)$ também apresentaram, aproximadamente, $51 \%$ mais chances de baixo índice de qualidade da dieta. Além disso, o nível socioeconômico mais baixo ( $\mathrm{p}=0,015$; OR=1,700; IC95\%=1,106 - 2,611) também esteve associado à dieta de qualidade intermediária.

Quanto à escolaridade materna, observa-se que os escolares cujas mães relataram menos do que três anos de estudo ( $\mathrm{p}=0,004 ; \mathrm{OR}=2,979 ; \mathrm{IC} 95 \%=1,423-6,239)$ apresentaram quase três vezes mais chances de apresentar baixa qualidade da dieta; e aqueles cujas mães relataram nível intermediário de estudo ( $\mathrm{p}, 0,001 ; \mathrm{OR}=1,658$; IC95\%=1,252 - 2,194) tiveram incremento de, aproximadamente, $66 \%$ nas chances de apresentar baixo índice dietético. Portanto, quanto menor 
o número de anos de estudo concluídos pela mãe, maior a chance de a criança apresentar dieta de baixa qualidade.

Sexo, idade, estado nutricional, região de residência e preocupação com o peso do filho não estiveram associados à qualidade da alimentação de escolares e, portanto, não foram incluídos no modelo de regressão logística.

\section{Discussão}

Revisão sistemática conduzida por Arpini et al. ${ }^{8}$ mostra que a percepção materna do peso corporal do filho está associada às práticas de controle alimentar infantil, principalmente quanto à restrição e/ou pressão alimentar, entretanto, sem relatos da literatura científica de tal associação com a qualidade da alimentação da criança. No presente estudo, em que se avaliou a qualidade global da dieta, os resultados evidenciaram que a concordância da percepção materna com o diagnóstico nutricional infantil não apresentou associação significante com a qualidade da dieta consumida pelos escolares. As características socioeconômicas foram as variáveis que impactaram no índice de qualidade global da dieta do escolar.

Em ambas as regiões, os escolares apresentaram maiores percentuais de baixa qualidade da dieta. Imagina-se que em um município rural cuja economia é baseada em produção de hortifrutigranjeiros, alimentos considerados "saudáveis" e marcadores de uma dieta de boa qualidade sejam consumidos em maior quantidade, enquanto numa região urbanizada, o maior acesso aos alimentos industrializados, dentre outros fatores, impactaria em maior prevalência de baixa qualidade da dieta. Contudo, os dados expressam semelhanças entre as duas populações, de maneira que ambas apresentaram maiores percentuais de baixa qualidade da dieta, ainda que na região urbana esse valor seja ligeiramente maior (40,4\% versus 38,6\%), conforme a tabela 1 .

Estudo de Lourenço ${ }^{17}$ realizado com famílias agricultoras do Rio de Janeiro mostrou que, para essa população, a produção agrícola objetiva a venda; plantar para autoconsumo é considerado perda de tempo, principalmente quando comparado à praticidade da compra dos alimentos e a valorização de produtos comercializados em detrimento dos produzidos. Em adição, a melhora da infraestrutura para acesso aos alimentos, como energia elétrica, transporte e mercados locais, pode estar favorecendo o consumo desses alimentos industrializados na região rural. ${ }^{17}$

De fato, em pesquisa qualitativa realizada com as mães dos escolares da região rural deste estudo, observou-se que a alimentação desta população está relacionada à rotina de trabalho dos adultos, bem como com as preferências alimentares da família e a disponibilidade de alimentos. ${ }^{12} \mathrm{~A}$ alimentação é baseada em pão ou brote (alimento tradicional da cultura pomerana) com margarina ou banha animal, nos lanches; e de arroz, feijão, carne, farinha e salada, nas principais refeições. ${ }^{12}$ Padrão alimentar semelhante foi descrito por Bezerra et al. ${ }^{18}$ nas regiões rurais brasileiras, e por 
Lourenço ${ }^{17}$ que, aponta ainda, o trabalho na agricultura e a pequena variedade de hortaliças cultivadas como possíveis fatores para o desestímulo ao consumo do que é produzido pelos agricultores. Desse modo, deve-se considerar que a alimentação está relacionada ao contexto sociocultural dos indivíduos e não deve ser tratada somente como uma questão biológica. ${ }^{12}$

Ainda que as mães das duas regiões estudadas tenham apresentado maiores percentuais de concordância e de preocupação com o peso do filho, aquelas residentes em município rural apresentaram maior concordância na percepção do estado nutricional do filho, enquanto que as do meio urbano apresentaram maior preocupação com o peso da criança. Estudo de Kobarg \& Vieira ${ }^{19}$ evidencia que crenças e práticas de cuidado materno podem ser diferentes em função do contexto em que as mães residem. As mães da região rural costumam disponibilizar mais tempo para a criação dos filhos e valorizar mais a disciplina, enquanto que as mães residentes em centros urbanos, devido à maior possibilidade de acesso à formação educacional e profissional, apresentam aumento da preocupação com relação a seus filhos.

É possível que a escolaridade materna também possa influenciar na qualidade da alimentação infantil, por meio das escolhas alimentares e do conhecimento ou discernimento das informações sobre nutrição obtidas, principalmente, por meio da mídia.,

Neste estudo, o fato de a mãe ser analfabeta ( $<3$ anos de estudo) aumentou, aproximadamente, três vezes a chance de a criança ter uma dieta de baixa qualidade, e esse risco diminuiu com o aumento da escolaridade. Em estudo nacional, a baixa escolaridade materna esteve associada ao consumo regular de refrigerantes, guloseimas, biscoitos doces e embutidos, e o aumento da escolaridade materna propiciou aumento do consumo de hortaliças por adolescentes. ${ }^{3}$

Outro fator associado à qualidade da dieta dos escolares desta população foi a raça/cor do escolar. As crianças pretas/pardas apresentaram aumento de $40 \%$ na probabilidade de ter uma dieta de baixa qualidade. A variável "raça/cor" pode ser considerada um marcador socioeconômico neste estudo, uma vez que em investigação conduzida nos escolares da região urbana (população predominante) esteve positivamente associada com a escolaridade materna e condição socioeconômica. ${ }^{20}$

A renda familiar, por sua vez, também pode dificultar o acesso a uma alimentação saudável..$^{21}$ A Pesquisa Nacional de Saúde do Escolar (PeNSE) ${ }^{3}$ evidenciou que o baixo nível socioeconômico aumenta a probabilidade do consumo de alimentos considerados "não saudáveis" e o melhor nível socioeconômico reflete um aumento da frequência do consumo de frutas e hortaliças por escolares.

Por outro lado, deve-se considerar que o aumento da renda pode contribuir para o aumento da frequência do consumo de alimentos fora do domicílio, principalmente na área urbana, com predominância dos alimentos de alta densidade calórica e pobre em nutrientes, em ambas as regiões (urbana e rural), ${ }^{18}$ refletindo numa alimentação de baixa qualidade. Em consonância, os resultados de investigação conduzida com escolares de sete a dez anos de idade em Florianópolis- 
SC mostraram que o aumento da renda familiar diminuiu a chance dos escolares de atender às recomendações para frutas e hortaliças. ${ }^{6}$

Neste estudo, os níveis socioeconômicos mais baixos aumentaram a probabilidade de o escolar apresentar menores escores de qualidade da dieta, resultado coerente com o encontrado por Lazarou \& Newby ${ }^{22}$ ao revisarem os estudos realizados nos países desenvolvidos sobre qualidade da dieta infantil.

Além disso, outras considerações podem ser feitas na compreensão dos fatores associados à qualidade da dieta infantil. A alimentação da criança pode ser influenciada pelos hábitos alimentares dos adultos. ${ }^{1}$ Quando os adultos apresentam dieta adequada às recomendações, dobra a probabilidade de a alimentação infantil também atender às recomendações. ${ }^{23}$ As crianças também são mais propensas a comer alimentos que estão disponíveis - então se maior quantidade é ofertada, a ingestão alimentar tende a ser maior. ${ }^{21}$ Outro fator é que os pais costumam querer agradar seus filhos e, numa forma de garantir que consumam alimentos saudáveis, oferecem guloseimas como recompensa. ${ }^{1}$

Inúmeros estudos evidenciam um padrão alimentar brasileiro caracterizado por frequente consumo de alimentos de baixo teor nutricional e consumo insuficiente de frutas e hortaliças, principalmente por crianças e adolescentes, ${ }^{3,4,6,18,24,25}$ alertando para a necessidade de intervenções para melhoria da qualidade da dieta, com incentivo de práticas alimentares saudáveis precocemente.

Embora o presente estudo evidencie resultados consistentes para o conhecimento sobre os fatores preditores da qualidade da dieta de escolares, o delineamento transversal nos impede de fazer inferência causal; todavia, é adequado aos objetivos deste estudo, por ser eficaz para a descrição das características da população e para identificação dos fatores associados.

Outra possível limitação do estudo refere-se à descrição verbal das mães quanto ao estado nutricional que percebem que seus filhos apresentam, utilizada para obtenção da variável "concordância da percepção materna”. Este é um método subjetivo, mas aceito e vastamente utilizado na literatura, por ser coerente com a abordagem do tema. O método utilizado para mensurar o consumo alimentar é limitado para avaliação da ingestão da dieta habitual, mas permite estimar a qualidade global da dieta do escolar por meio da frequência do consumo de alimentos.

A comparação dos resultados do presente estudo com outros é limitada devido às diferenças existentes nas populações, dos instrumentos e métodos de avaliação dietética utilizados e das variáveis associadas. Apesar das limitações descritas, os resultados aqui apresentados são coerentes com outros estudos transversais e longitudinais. Ademais, o mesmo pode ser útil como base para novas investigações.

Assim, outros estudos são necessários para investigar os fatores relacionados à qualidade da alimentação em diferentes populações, inclusive quanto à relação com a percepção materna do 
estado nutricional do filho, e que possibilite a compreensão desses fatores nos diferentes contextos urbano e rural.

\section{Conclusão}

Não foi encontrada associação entre a alimentação infantil e a concordância da percepção materna com o diagnóstico do estado nutricional. Todavia, os determinantes socioeconômicos influenciaram diretamente na qualidade da dieta dos escolares.

\section{Referências}

1. Adamo KB, Brett KE. Parental perceptions and childhood dietary quality. Matern. Child Health J. 2014; 18(4):978-95.

2. Kranz S, Findeis JL, Shrestha SS. Use of the Revised Children's Diet Quality Index to assess preschooler's diet quality, its sociodemographic predictors, and its association with body weight status. J. Pediatr. (Rio J). 2008; 84(1):26-34.

3. Levy RB, Castro IRR, Cardoso LO, Tavares LF, Sardinha LMV, Gomes FS, et al. Food consumption and eating behavior among Brazilian adolescents: National Adolescent School-based Health Survey (PeNSE), 2009. Ciênc. Saúde Coletiva 2010; 15(Supl. 2):3085-97.

4. Coelho LG, Cândido APC, Machado-Coelho GLL, Freitas SN. Association between nutritional status, food habits and physical activity level in schoolchildren. J. Pediatr. (Rio J). 2012; 88(5):406-12.

5. Molina MCB, Lopéz PM, Faria CP, Cade NV, Zandonade E. Preditores socioeconômicos da qualidade da alimentação de crianças. Rev. Saúde Pública 2010; 44(5):785-732.

6. Assis MAA, Calvo MCM, Kupek E, Vasconcelos FAG, Campos VC, Machado M, et al. Qualitative analysis of the diet of a probabilistic sample of schoolchildren from Florianópolis, Santa Catarina State, Brazil, using the Previous Day Food Questionnaire. Cad. Saúde Pública 2010; 26(7):1355-65.

7. Ambrosini GL, Emmett PM, Northstone K, Howe LD, Tilling K, Jebb SA. Identification of a dietary pattern prospectively associated with increased adiposity during. childhood and adolescence. Int. J. Obes. 2012; 36(10):1299-305.

8. Arpini LSB, Queiroz DMF, Corrêa MM, Salaroli LB, Molina MCB. Relação entre a percepção materna do peso corporal do filho e as práticas alimentares infantis. Rev Bras Pesq Saúde. 2014; 16(3):140-153.

9. Manios Y, Moschonis G, Grammatikaki E, Anastasiadou A, Liarigkovinos T. Determinants of Childhood Obesity and Association with maternal perceptions of their children's weight status: The “GENESIS” Study. J. Am. Diet Assoc. 2010; 110(10):1527-31.

10. Faria CP, Cade NV, Nascimento LR, Molina MCB. Prevalência de excesso de peso em crianças de sete a dez anos de Vitória-ES, Brasil: um estudo de base escolar. Rev. Bras. Pesqui. Saúde 2011; 13(1):31-7. 
11. Justo GF, Callo GQ, Carletti L, Molina MCB. Nutritional extremes among school children in a rural Brazilian municipality. Rural Remote Health 2012; 12(4):2220.

12. Justo GF, Callo GQ, Molina MCB, Cade NV. Hábitos alimentares entre mães de escolares residentes em um município rural do Espírito Santo: um estudo observacional. Rev. Bras. Pesq. Saúde 2011; 13(3):17-21.

13. World Health Organization. The international classification of adult underweight, overweight and obesity according to BMI. Geneva: WHO; 2004.

14. Associação Brasileira de Empresas de Pesquisa. Critério de classificação econômica Brasil [Internet]. 2010 Acesso em: 20 mar. 2014. Disponível em: http://www.abep.org/criterioBrasil.aspx

15. Onis M, Onyango AW, Borghi E, Siyam A, Nishida C, Siekmann J. WHO Development of a WHO growth reference for school-aged children and adolescents. Bulletin of the World Health Organization 2007; 85(9):660-667.

16. World Health Organization. Physical status: the use and interpretation of anthropometry indicators of nutritional status. Geneva: WHO; 1995. Report No. 854.

17. Lourenço AEP. Plantando, colhendo, vendendo, mas não comendo: práticas alimentares e de trabalho associadas à obesidade em agricultores familiares do Bonfim, Petrópolis, RJ. Rev. Bras. Saúde Ocupacional 2012; 37(125):127-42.

18. Bezerra IN, Souza AM, Pereira RA, Sichieri R. Consumption of foods away from home in Brazil. Rev. Saúde Pública 2013; 47:200s-211s.

19. Kobarg APR, Vieira ML. Mothers' beliefs and practices about child development in rural and urban contexts. Psicol. Reflex. Crítica 2008; 21(3):401-8.

20. Molina MCB, Faria CP, Montero P, Cade NV. Correspondence between children's nutritional status and mothers' perceptions: a population-based study. Cad. Saúde Pública 2009; 25(10):2285-90.

21. Patrick H, Nicklas TA. A review of family and social determinants of children's eating patterns and diet quality. J. Am. Coll. Nutr. 2005; 24(2):83-92.

22. Lazarou C, Newby PK. Use of dietary indexes among children in developed countries. Adv. Nutr. Int. Rev. J. 2011; 2(4):295-303.

23. Zuercher JL, Wagstaff DA, Kranz S. Associations of food group and nutrient intake, diet quality, and meal sizes between adults and children in the same household: a cross-sectional analysis of U.S. households. Nutr J. 2011; 10:131.

24. Costa LCF, Vasconcelos FAG, Corso ACT. Factors associated with adequate fruit and vegetable intake by schoolchildren in Santa Catarina State, Brazil. Cad. Saúde Pública 2012; 28(6):1133-42.

25. Souza AM, Pereira RA, Yokoo EM, Levy RB, Sichieri R. Most consumed foods in Brazil: National Dietary Survey 2008-2009. Rev. Saúde Pública 2013; 47:190s-199s.

Recebido: 30/1/2015

Revisado: $16 / 3 / 2015$

Aprovado: 06/4/2015 